\title{
Intersubjetividad, empatía y educación. Una aproximación fenomenológica
}

\author{
Rubén Sánchez Muñoz \\ Universidad Popular Autónoma del Estado de Puebla \\ ruben.sanchez.munoz@upaep.mx \\ https://orcid.org/0000-0002-3010-5810
}

Resumen: El objetivo de este trabajo es describir desde una perspectiva fenomenológica: a) las relaciones entre la intersubjetividad y el fenómeno de la educación; b) la empatía como base y fundamento de las relaciones intersubjetivas; y c) la importancia de la empatía para la educación. La tesis que se defenderá sostiene que todo proceso educativo de enseñanza-aprendizaje, sea en la escuela, sea en la familia o en la sociedad, necesita de la experiencia de los otros, cuestión posibilitada por la empatía. Esta experiencia está mediada por el cuerpo y se desarrolla en una dimensión intercultural, intersubjetiva e intercorpórea.

Palabras clave: empatía; intersubjetividad; cuerpo; experiencia; educación

\begin{abstract}
Intersubjectivity, Empathy and Education. A Phenomenological Approach". The aim of this paper is to describe from a phenomenological perspective: a) the relationships between intersubjectivity and the phenomenon of education; b) empathy as the basis and the foundation of intersubjective relationships; and c) the importance of empathy for education. The thesis to be defended states that every educational process of teaching and learning, whether in school, in the family or in society, needs the experiencing of others and empathy makes it possible to access this sort of experience. This experience is mediated by the body and develops into an intercultural, intersubjective and inter-corporeal dimension. Keywords: empathy; intersubjectivity; body; experience; education
\end{abstract}




\section{Introducción}

¿Qué lugar ocupa la empatía (Einfühlung) dentro de la educación? Los apartados en los que se divide este artículo de investigación serán los siguientes: a) marcar la relación intersubjetiva en la que se inscribe el proceso de educación; b) mostrar que las bases de estas relaciones intersubjetivas se hallan en el fenómeno de la empatía, para lo cual es necesario presentar una definición y algunos aspectos fundamentales de la empatía, y c) describir algunas notas esenciales del lugar que ocupa la empatía para la educación. La tesis que vamos a sostener es la siguiente: todo proceso educativo toma como base o punto de partida la experiencia que tenemos de los otros y la empatía es el fenómeno que nos permite acceder a la experiencia ajena. Lo que está presuponiéndose en el fenómeno de la empatía es que hay en el mundo otros sujetos yo, el alter ego, y que el yo puede acceder de algún modo a sus vivencias. Sin embargo, ¿cómo debemos entender la empatía? El enfoque a partir del cual vamos a realizar este trabajo es el fenomenológico. Seguiremos para ello las indicaciones de Edith Stein y Edmund Husserl, principalmente, y nos apoyaremos en algunas investigaciones fenomenológicas recientes sobre el fenómeno de la educación.

\section{Educación e intersubjetividad}

La educación apunta, por su propia naturaleza, a una dimensión fundamental de la persona, a saber, su dimensión social. Esta socialidad de la persona no es un añadido, ni un aspecto que se pueda tener o no, sino una dimensión esencial que se presenta en distintos niveles que pueden diferenciarse. Están, por ejemplo, la comunidad, la sociedad, el pueblo, la masa, el Estado, y en ellas la persona despliega su vida. En todo caso, la vida se vive intersubjetivamente. Lo que la persona llega a ser, llega a serlo en sociedad. Se vive entre sujetos, junto a ellos, rodeados de ellos, en ellos, por y para ellos, entregados a ellos, huyendo o cuidándose de ellos, etcétera. La vida personal, stricto sensu, es vida con otros. Estos otros están en el horizonte de nuestra vida ya sea de modo latente, ya sea de modo patente. Además, están en relación de maneras distintas y muy diversas.

¿De qué modo me relaciono con los otros? ¿Quiénes son los otros? El otro es mi alter ego, el yo que no soy yo y el cual, sin embargo, es como yo en 
muchos sentidos: es otro yo. No obstante, ¿cómo tengo notica del alter ego? De acuerdo con la tradición fenomenológica, cuyo enfoque seguimos en este trabajo, el otro no se me da o no tengo experiencia de él solo en percepción externa (por ejemplo, en el darse del cuerpo), sino que hay otros actos de conciencia en los cuales el otro se da. Este, por cierto, es uno de los problemas fundamentales de la filosofia moderna y contemporánea: ¿cómo conocemos a los otros? Empezamos haciendo énfasis en la dimensión intersubjetiva de la persona, justamente porque la educación es un fenómeno que solo tiene sentido y razón de ser en la relación de unos con otros y en el reconocimiento mutuo. Por ello, tanto la intersubjetividad como la alteridad se refieren a esta pluralidad de sujetos-yo que se relacionan entre sí. Así, la posibilidad de un individuo aislado, fuera de la sociedad, es un engaño, porque esencialmente el ser humano es un ser gregario. Pero esta pluralidad de sujetos, como vamos a ver en las páginas siguientes, da pie para hablar también de una pluralidad de mundos subjetivo-relativos que aparecen mediando o determinando, unas veces a favor, otras más en contra de, las relaciones intersubjetivas.

Estamos en relación con los otros antes del nacimiento y seguimos en contacto con los otros incluso más allá del tiempo y de la muerte, esto es: la persona que muere sigue presente de muchas maneras en la vida de los individuos que estuvieron en contacto con ella. La relación con el otro no se da solo y de manera exclusiva en el presente, sino que "respecto del pasado se da por

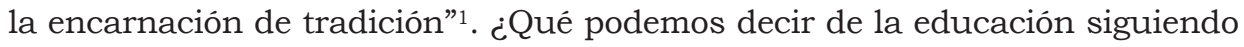
estas indicaciones? La educación, sostiene Vincenzo Costa, "es un proceso mediante el cual un ser humano abre a otro ser humano a las propias posibilidades, despliega ante él el horizonte de lo posible y le abre a la comprensión del mundo y de sí mismo, de modo que sea él quien pueda ejercitar la propia libertad"2. Con ello puede verse que se trata de un acto intersubjetivo, de un acto en el que se dan cita varios actores o sujetos. "La educación constituye la tarea mediante la cual la persona es puesta ante sí, ante el propio poder ser en orden a definir cómo quiere usar ese tiempo que es y cómo se va a relacionar con las posibilidades que le ofrece su época"3.

De acuerdo con esta definición, en la educación "un ser humano abre a otro ser humano a las propias posibilidades". Pensemos, por consiguiente,

\footnotetext{
1 Iribarne, J., En torno al sentido de la vida. Ensayos fenomenológicos sobre la existencia, Morelia: Jitanjáfora, 2012, p. 175.

2 Costa, V., Fenomenología de la educación y la formación, Salamanca: Sígueme, 2018, p. 76.

3 Ibid., p. 91. El énfasis es del autor.
} 
que a) un individuo aislado es imposible y que b) nadie se educa solo, sino que necesita que alguien lo acompañe y le muestre o abra esas posibilidades. En este sentido, es razonable pensar en el sujeto de la educación como un sujeto de potencialidades que debe actualizar y desplegar en el curso de su existencia. Pero, ¿sabe el educador de esos potenciales o más bien tiene él mismo que descubrirlos junto con el educando? No es irracional sostener, sobre este punto, que en todo proceso de educación ambos, tanto educador como educando, se alteran mutuamente, o sea, se modifican. Por ello, en el horizonte de la intersubjetividad, son los sujetos que participan en la educación quienes se abren a lo posible y se comprenden como sujetos. En tanto que en la educación los sujetos salen al encuentro con aquello que, desde el inicio, les es desconocido, puede decirse, entonces, que se ven alterados. Es en el encuentro intersubjetivo y en el encuentro con la realidad que lo circunda donde el sujeto se reconoce como sujeto capaz o, en su defecto, donde se reconoce como sujeto incapaz. Para el caso que nos ocupa, nos interesa el sujeto de la educación en tanto que es un sujeto de capacidades ${ }^{4}$. Sin embargo, estas capacidades deben reconocerse: las debe identificar y reconocer el educador y, del mismo modo, las debe identificar y reconocer para sí mismo el educando.

Sobre este punto, J. Vlieghe nos recuerda lo importante que eran para John Dewey las experiencias vividas del mundo como punto de partida para el aprendizaje, el poder hacer, manipular, vivir la experiencia por parte del niño, pero, especialmente, que el aprendizaje no se da nunca en solitario, sino que se aprende en presencia de otros; en consecuencia, "toda actividad educativa es potencialmente una situación de cooperación”. Paulo Freire enfatizó en ello también al decir que nadie educa a nadie y también que nadie se educa solo, más aun: que los hombres se educan entre sí ${ }^{6}$.

Esta es la razón que justifica el sentido etimológico de la palabra educación: educar viene del latín educare, el cual significa "criar, cuidar, alimentar o informar o instruir" y de educere: "sacar o extraer, avanzar, elevar"; ducere, por

\footnotetext{
4 En este trabajo no seguimos el planteamiento de Martha Nussbaum sobre el desarrollo de las capacidades, pero es una obra que debe tenerse en el marco de referencia para el tema que nos ocupa. Nussbaum se refiere a las capacidades como aquello "que es capaz de hacer y de ser una persona". Cf. Nussbaum, M., Crear capacidades: propuestas para el desarrollo humano, Barcelona: Paidós, 2012, p. 38.

5 Vlieghe, J., "The Body in Education", en: Paul Smeyers (ed.), International Handbook of Philosophy of Education, v. II, Nueva York: Springer, 2018, p. 1018.

6 Barreiro, J., "Educación y concienciación", en: Freire, P. La educación como práctica de la libertad, México: Siglo XXI, 2020, p. 19.
} 
su parte, significa "conducir" y, en la voz pasiva, "crecer"7. En esta pluralidad de significados se dejan ver las distintas dimensiones en las que opera el fenómeno educativo. La educación, así, engloba como tal una serie de "dimensiones operativas" en las que están en juego diversos elementos y es tan compleja como la realidad y el ser mismo del ser humano.

Lo que hay que decir, entonces, siguiendo a Costa, es que, en tanto que es un proceso que consiste en "conducir", la educación involucra la acción o participación intersubjetiva, la colaboración de varios sujetos dentro de los cuales unos nutren, informan o instruyen a otros; o quizás sea mejor decir que la educación es un proceso mediante el cual los actores o sujetos se nutren, se informan y se instruyen unos a otros en múltiples direcciones. No podríamos decir que, en la escuela o en la familia, solo los alumnos o los hijos aprenden de los maestros o los padres. Por el contrario, también los maestros y los padres aprenden de los estudiantes y los hijos. En esta compleja relación, todos se nutren de todos, pues están en relación reciproca. ¿Qué condición hace posible esta modificación de unos por otros?

Es un lugar común referirse a la intersubjetividad como alteridad. La alteración es, en efecto, lo que ocurre cuando salimos al encuentro del otro y nos dejamos interpelar por él. El otro o lo otro (y este puede ser otro yo o bien la realidad dada como otro) nos altera - como expone Ortega en Ensimismamiento $y$ alteración ${ }^{8}$ - . El otro nos interpela y lo hace de muchas maneras. Como ha expuesto Levinas, el alter ego, el otro, nos modifica, exige de nosotros una respuesta, nos solicita. El otro yo supone o conduce a pensar en la ética y ello se deja ver claramente en la obra de Levinas, quien sostiene, en Ética e infinito, que la relación con el otro es una relación ética que demanda una respuesta del yo al llamado del otro ${ }^{\cdot}$ No obstante, cabe decir que, más allá del solipsismo metodológico de Husserl como aparece en la quinta meditación cartesiana, la reflexión sobre el otro lo lleva al planteamiento de una ética universal. De acuerdo con Husserl: "la reflexión ética universal no solo me concierne a mí... Mi auto-responsabilidad incluye una responsabilidad por el ser de los otros en la razón práctica... El ser-con otro del ser humano es un comunicar, un vivir en común... Todos son responsables de todos. No existe una mera ética

\footnotetext{
7 Altarejos, F. y C. Naval, Filosofia de la educación, Pamplona: EUNSA, 2011, p. 20ss.

8 Ortega y Gasset, J. "Ensimismamiento y alteración”, en: Obras completas, v., Madrid: Taurus / Fundación José Ortega y Gasset-Gregorio Marañón, pp. 529-550.

9 Levinas, E., Ética e infinito, Madrid: Machado Libros, 1991, caps. 7 y 8.
} 
privada, sino que la ética individual y la social, la ética universal humana, son todas una ética"10.

Ello solo es posible entre sujetos que se dejan determinar unos a otros, que se motivan o bien se obstruyen y obstaculizan. La vida interpersonal es vida en común (en común unidad, o sea, en comunidad). El mundo se vive intersubjetivamente, entre sujetos que se alteran y modifican unos a otros. Pero que unos influyan en otros supone o exhibe un elemento fundamental de las relaciones humanas: la apertura de unos a otros. Que haya una apertura deja abierta la posibilidad de que eventualmente haya también una cerrazón $n^{11}$.

Esto es lo que ocurre esencialmente en la educación. Unos sujetos conducen a otros y los abren a nuevas posibilidades de acción, de existencia. Sin embargo, este es un intercambio mutuo. Si es simétrico o no, eso no lo sabemos. Quizás importe poco. Lo que realmente importa es que, en este encuentro interpersonal, nadie se queda en el sitio donde empezó. Cada uno se ve alterado o interpelado por la presencia del otro. Porque las cosas son así, es que nos preguntamos: ¿qué lugar ocupa la empatía en la educación y qué relación tiene la empatía con la intersubjetividad?

En Fenomenología de la educación y la formación, Costa afirma que "el sentido de la educación y de la formación reside en la continua reapertura del sujeto a lo posible y, por tanto, al mundo como conjunto de posibilidades, al tiempo como tener que ser y a los otros como seres con los que estamos juntos en el mundo"12. Esta indicación nos parece valiosa porque apunta a varias dimensiones de la realidad personal: (1) la apertura o reapertura del sujeto al mundo; (2) la dimensión temporal y, por tanto, dinámica del ser personal; y (3) la constitución intersubjetiva del mundo. Ahora bien, el mundo no es una realidad dada sin más, no es la totalidad de las cosas que hay, sino el conjunto de relaciones y significados en las que vive y con las que está en relación la persona. En efecto, "el mundo no es una cosa enorme o un conjunto de cosas, sino el sistema de referencias que las hace significantes... El mundo es, por tanto, un sistema de diferencias y referencias en que todo elemento tiene un cierto significado en virtud de su posición dentro del sistema global"13. Ese mundo no

${ }^{10}$ Véase: Ms. A V 22, pp. 12a-13a. Citado en: Rizo-Patrón, R., El exilio del sujeto. Mitos modernos y posmodernos, Lima: Aula de Humanidades/Fondo Editorial de la Pontificia Universidad Católica del Perú, 2014, p. 431.

${ }^{11}$ Cf. Stein, E., Der Aufbau der menschlichen Person. Vorlesung zur philosophischen Antropologie,

14 , Friburgo: Herder, 2004.

${ }^{12}$ Costa, V., Fenomenología de la educación y la formación, p. 198.

${ }^{13}$ Ibid., p. 100. El subrayado es del autor. 
se constituye o llega a tener el sentido de manera solipsista e individual, sino a través de las relaciones intersubjetivas. Llegamos a un mundo donde ya están operando sistemas de referencias, valores, usos, costumbres, tradiciones, en términos amplios: cultura.

Vale la pena preguntar cómo hay que entender la cultura. Dice Edith Stein que la cultura es "...todo cosmos de bienes espirituales unitario en sí y deslindando de cara a fuera (trátese tanto de objetos consistentes u obras de arte y de la ciencia, como de formas de vida estilizadas predominantes en la vida actual de las personas)"14. Pero, además, argumenta que la cultura “... remite a un centro espiritual al que ellos deben su origen; y este centro es una comunidad creadora cuya peculiaridad anímica específica se traduce y refleja en todas sus producciones"15.

Por su parte, Husserl sostuvo que la vida propiamente humana es vida en la cultura. Definió la cultura como “...el conjunto total de logros que vienen a la realidad merced a las actividades incesantes de los hombres en sociedad y que tienen una existencia espiritual duradera en la unidad de conciencia colectiva y de la tradición que la conserva y prolonga"16. La cultura, en consecuencia, es el resultado del trabajo de los hombres que viven unos con otros y unos en los otros. El aporte de unos individuos a la cultura se prolonga y se mantiene de una época a otra, de una generación a otra. Se vive, entonces, en un horizonte cultural, en un mundo intersubjetivo, cuya intersubjetividad está presente y manifiesta de manera explícita o implícita. La intersubjetividad se despliega en el horizonte cultural, en el que unos individuos son educados por otros. ¿Qué valor tiene la cultura para la educación? No se puede educar, en el sentido que sea, al margen de la cultura. Más bien, la educación es el proceso mediante el cual el individuo es introducido en la cultura, esto es, en el sistema de valores que constituyen el horizonte en el que se despliega la vida de los sujetos que viven juntos, unos más cerca, otros más lejos. En consecuencia, el sujeto que se educa se cultiva, se inserta en la cultura que le rodea, empezando por la más próxima (que es la cultura en la que vive y conforma su mundo familiar) y, poco a poco, extendiendo los círculos concéntricos más allá, hacia el exterior (hacia el mundo extraño, el que originariamente le es ajeno). La cultura, a juicio de

\footnotetext{
14 Stein, E., Una investigación sobre el Estado, Madrid: Trotta, 2019, p. 29.

15 Ibid., p. 29.

${ }^{16}$ Husserl, E., Renovación del hombre y de la cultura, Barcelona/México: Anthropos/UAM, 2002, p. 22 .
} 
Husserl, "posee un tipo esencialmente peculiar de existencia objetiva, y donde opera, por otra parte, como una fuente permanente de socialización"17.

En efecto, la educación se da al interior de cada cultura y "nos ayuda paulatinamente a superar toda suerte de incomprensibilidades iniciales, como cuando aprendemos a leer, a calcular, a apreciar o tocar una pieza musical.. [porque] esto ya ocurre en todo aprendizaje de artes o disciplinas científicas y culturales en un 'mundo familiar' o cultura dada. Se trata de una expansión de lo familiar a lo menos familiar dentro del propio 'mundo familiar"'18.

Digamos, entonces, que el sujeto de la educación (y este no es propiamente el estudiante, sino ambos, tanto estudiante como docente), se encuentra en un mundo familiar, en un mundo de la vida que originariamente le es dado desde el nacimiento, pero el cual se le presenta con "toda suerte de incomprensibilidades iniciales que tiene que superar". Y las supera con la ayuda y la guía de los otros, de los que llegaron antes e incluso de los que ya no están. Entre esos otros hay quienes tienen mayor relación con el mundo, disponen de un horizonte más amplio de sentido y viven en una relación más estrecha con los significados que el mundo en torno les ofrece. La educación, en consecuencia, hace posible el encuentro de lo que hay en el mundo familiar del sujeto con lo que hay el mundo extraño. Dentro de la multiplicidad de acepciones que se pueden emplear, puede decirse que lo extraño es lo ajeno, lo que no es de mi propiedad y también lo que es desconocido para mi $^{19}$. Pero lo que es desconocido para mí es conocido por otro. El mundo extraño para mí es mundo familiar para otros. Digamos, entonces, que en la educación se da un encuentro con lo extraño y esto "acarrea simultáneamente un cambio en la actitud en la relación con el propio "mundo familiar"'20.

En el ámbito de la intersubjetividad social o bien en el de las comunidades humanas, se da una relación en la que sus "miembros individuales se hallan recíprocamente orientados unos a otros"; esto es, la comunidad se caracteriza por los actos sociales mediante los cuales se da la comunicación humana. Esta comunicación constituye el acto original de la relación yo-tú. "La reciprocidad de los actos sociales -de acuerdo con Rizo-Patrón- se efectiviza, pues, tanto en el estar orientado perceptivamente el uno al otro, mirándose a los ojos, el

\footnotetext{
17 Ibid., p. 22.

${ }^{18}$ Rizo-Patrón, R., El exilio del sujeto. Mitos modernos y posmodernos, p. 316.

${ }^{19}$ Cf. Bernhard, W., Exploraciones fenomenológicas acerca de lo extraño, Barcelona/Morelia: Anthropos/UMSNH, , 2015.

20 Rizo-Patrón, R., El exilio del sujeto, p. 316.
} 
estar consciente el uno del otro, tocándose espiritualmente, como también en el discurso directo, como saludando, hablando, escuchando, respondiendo, etc.". ${ }^{21} \mathrm{El}$ mundo en que vivimos es un mundo interpersonal en el que nos determinamos unos a otros y en la escuela, en la familia y en general en todos los grupos sociales a los que pertenecemos y en los cuales somos educados y educamos, vemos cómo las vidas, los deseos y aspiraciones de unos se convierten en vidas, deseos y aspiraciones de los otros.

Esto que acabamos de describir es lo que se da en la educación. La razón de ello es que la educación se desarrolla en el horizonte de la intersubjetividad, a través de actos sociales que se fundan en la comunicación de unos con otros, en las respuestas, el guiar y llevar, el orientar y el "tocar espiritualmente" unos a otros. La educación parte de una precariedad originaria de la persona o sujeto que se educa, del estar en el mundo y tener que superar ciertas zonas que le resultan incomprensibles del mundo mismo y del propio yo, de la personalidad propia, que poco a poco va esclareciendo, viendo nuevas relaciones de sentido $\mathrm{y}$, con ello, ampliando sus horizontes vitales. Por ello, como dice Costa, en la educación la persona es puesta ante sí, pero nunca de manera aislada o dando la espalda al mundo o circunstancia, sino que la persona está puesta ya en ese mundo de manera originaria y, además, en un mundo cultural que remite por todas partes a los otros. El mundo se constituye intersubjetivamente, es lo que es en la relación de unos con otros ${ }^{22}$.

La educación, en efecto, "no es un espacio aislado, sino una parte constitutiva de la cultura más amplia de una sociedad... La educación y la sociedad están en continuo intercambio; es una ingenuidad perniciosa asumir que los cambios en el ámbito de la educación no tienen ningún efecto en la sociedad o viceversa"23.

No cabe duda que aspectos importantes dentro de la cultura en que se habita son la tradición y el modo o los modos cómo nos relacionamos con ella. Somos herederos de una tradición y en el proceso que se llama educación aprendemos o buscamos la manera de entrar en diálogo con ella y, por tanto,

\footnotetext{
21 Ibid., p. 310.

22 Véase: Zahavi, D., "Husserl's Intersubjective Transformation of Transcendental Philosophy", en: Welton, D. (ed), The New Husserl. A Critical Reader, Bloomington: Indiana University Press, 2003, pp. 233-251.

${ }^{23}$ Antoniou, K. y V. Karavakou, "Education in Crisis: Reflections on the Contribution of Phenomenology to Modern Educational and Political Culture”, en: Brinkmann, M. y otros, (eds.), Pädagogik-Phänomenologie. Verhältnisbestimmungen und Herausforderungen, v. III, Gewerbesrasse: Springer Nature, 2017, p. 220. Traducción nuestra.
} 
con los otros que hicieron posible las instituciones originarias que llegaron a prolongarse y extenderse, que se siguen cuidando, transmitiendo de unos a otros. En todo caso, el acto intersubjetivo que llamamos educación nos permite “...dialogar con la tradición, para la que la formación representa el itinerario mediante el cual todo recién llegado se inserta en la vivencia de un proceso de trasmisión histórica que es un proceso de auto-humanización y, en consecuencia, de toma de conciencia del propio formar parte de una humanidad que quiere configurarse a sí misma y el propio mundo circundante del modo más humano, formando 'un mundo humanamente bello y bueno"'24.

Empero, ¿qué quiere decir esta expresión según la cual el mundo se constituye intersubjetivamente? ¿Cómo tengo acceso a las representaciones del mundo que originariamente son ajenas? El fenómeno que nos permite comprender lo que venimos describiendo se llama empatía. ¿Qué es la empatía?

\section{Intersubjetividad y empatia}

El problema de la intersubjetividad, en el horizonte de la tradición fenomenológica, es identificado con el problema que supone el conocimiento de otras mentes ${ }^{25}$. ¿Cómo sabemos o podemos dar cuenta de que el otro piensa, siente, desea, quiere o bien sufre, le duele, etcétera? Si sensu estricto cada yo tiene acceso de modo originario a sus propios actos de conciencia, a lo que él mismo vive en primera persona, ¿cómo sabemos de las vivencias ajenas?

En la actualidad, quien mayormente ha trabajado el tema de la empatía desde el punto de vista de la percepción social, la intencionalidad y el conocimiento interpersonal en una perspectiva fenomenológica ha sido Dan Zahavi. Como señala Zahavi, "el enfoque empático se ha asumido ocasionalmente para constituir el enfoque fenomenológico de la intersubjetividad". La empatía, dice allí mismo, "entendida como un encuentro temático con un otro concreto se toma como un derivado en lugar de una forma fundamental de la intersubjetividad". ${ }^{26}$ En este trabajo, argumenta que hay aspectos de la intersubjetividad que no se pueden abordar si uno se centra en la empatía ${ }^{27}$. A su juicio, tanto en Husserl 346 como en Merleau-Ponty, encontramos que mi encuentro con el otro, mi habilidad para interactuar y reconocer la otra subjetividad encarnada como subjetividad

\footnotetext{
${ }^{24}$ Costa, V., Fenomenología de la educación y la formación, p. 66. Subrayado del autor.

${ }^{25}$ Zahavi, D., Subjectivity and Selfhood: Investigating the First-person Perspective, Cambridge, MA.: MIT University Press, 2006, p. 148ss.

${ }^{26}$ Ibid., p. 156.

${ }^{27}$ Ibid., p. 156.
} 
extraña son adelantados y hechos posibles a través de mi propia subjetividad encarnada ${ }^{28}$. Como veremos a continuación, esta misma idea está presente en la investigación fenomenológica sobre la empatía de Edith Stein. En efecto, en su libro The Phenomenological Mind, dedican Gallagher y Zahavi el Capítulo 9 a responder la pregunta: “¿Cómo conocemos a los otros?”. En él discuten la importancia de la empatía en relación con otros argumentos, como es el argumento de analogía o la simulación implícita ${ }^{29}$.

La empatía, de acuerdo con Edith Stein, es "la aprehensión de vivencias ajenas"30 ¿ ¿De qué vivencias ajenas se tiene aprehensión empatizante? Suele relacionarse la empatía con los sentimientos y las emociones, con el ponerse en el lugar del otro y sentir lo que él siente. Sin embargo, cabe decir que la empatía no se reduce a los sentimientos y emociones, sino que, en términos generales, aplica a cualquier vivencia de otros sujetos, incluyendo percepciones, recuerdos, sensaciones, etcétera. No se tiene empatía de un sujeto solo por sentir lo que siente, sino porque se puede acceder a sus campos visuales, táctiles, auditivos, olfativos o gustativos. Puede ser, entonces, que los sentimientos y las emociones jueguen un lugar especial, pero no por ser el campo o región exclusiva de la empatía, sino porque en ellos ocurre algo particular en la persona que experimenta la empatía y accede así a la vida afectiva del otro. ¿Qué es lo que ocurre en la primera persona que tiene la aprehensión de vivencias afectivas ajenas?

La empatía, en efecto, ocupa un lugar especial dentro de las recientes investigaciones sobre la cognición social. De acuerdo con Magrì, filósofos y psicólogos no se ponen de acuerdo en qué elementos son aquellos que aparecen en la empatía ${ }^{31}$. Además, considera que el método fenomenológico es importante para la descripción de la empatía, porque conecta el tema de la percepción con la expresividad corporal. El cuerpo vivo de la persona tiene la peculiaridad de ser un cuerpo expresivo, lo que hace, a su vez, que en esa expresividad se manifieste la unidad psicofísica de la persona. El cuerpo, entonces, ocupa un lugar importante para comprender el fenómeno de la empatía. En ella, la percepción que tenemos del otro no es igual a la percepción que tenemos de un objeto. La razón es que el otro no es un objeto, sino un sujeto como yo, si bien se trata de otro yo. Pero demos un paso atrás.

\footnotetext{
28 Ibid.

${ }^{29}$ Cf. Gallagher, S. y D. Zahavi, Phenomenological Mind, Londres: Routledge, 2020.

${ }^{30}$ Stein, E., Zum Problem der Einfühlung, ESGA 5, Friburgo: Herder, 2008, p. 78.

31 Magrì, E., "Subjectivity and Empathy: A Steinian Aproach", en: Mariani, E. (ed.), Discipline Filosofiche: Figures, Functions and Critique of Subjectivity beginning from Husserlian Phenomenology, v. XXV, 2 (2015), pp. 129-148
} 
Las lecciones de Husserl de 1910-1911 sobre Problemas fundamentales de la fenomenología contienen indicaciones de mucho valor para comprender qué es la empatía. Dejaremos a un lado las cuestiones relacionadas al problema del solipsismo y, en consecuencia, el problema que supone el conocimiento del otro. Lo que importa aquí es describir y presentar las bases que hacen posible la vivencia del otro y, en consecuencia, su conocimiento. ¿Qué dice el autor en esta obra?

Husserl habla de la empatía (Einfühlung) y del yo ajeno tomando como punto de partida la percepción externa del otro. "En ella el yo empatizante (das Einfühlende Ich) tiene experiencia de la vida anímica, o más precisamente, de la conciencia de otro yo"32. En efecto, el otro se aparece ante mí en primer lugar en la percepción corporal, pero veo que su aparición no es igual a como se aparece un objeto. El otro cuerpo es un cuerpo ajeno o extraño; es el cuerpo de "otro yo o un yo ajeno": "el yo mira esos cuerpos como 'portadores' de sujetos-yo, pero no 've' los yoes ajenos en el mismo sentido en que se ve o encuentra a sí mismo en su propia experiencia. Cada yo pone a esos otros yoes en el modo de la 'empatía' y con ello encuentra también las disposiciones de carácter y el vivenciar ajenos que, sin embargo, no son dados o tenidos como propios" ${ }^{3}$.

Al igual que el yo, el otro yo tiene un alma, disposiciones de carácter, conciencia actual, capacidades, etcétera, y está en un mundo que lo rodea: "el entorno que encuentra el yo ajeno, pero que está frente a nosotros casi perceptiblemente, sería en general y por completo el mismo que el nuestro" 34 . Con su cuerpo pasa lo mismo. Así como yo tengo experiencia originaria de mi cuerpo, lo vivo y experimento en primera persona, del mismo modo puede anticiparse que el cuerpo del otro es vivido de manera originaria como cuerpo propio. Se trata de un cuerpo propio ajeno, o sea, de otro. Así, “...lo válido para los entornos actuales de los yoes que se encuentran recíprocamente en el modo señalado y se incorporan recíprocamente en sus entornos, vale para el mundo en su totalidad. Todos esos yoes se captan como centros relativos al único y mismo mundo espaciotemporal, el entorno total (Gesamtumgebung) de todo yo. Para cada yo los otros yoes no son centros, sino puntos del entorno que tienen,

\footnotetext{
${ }^{32}$ Husserl, E., Problemas fundamentales de la fenomenología, Madrid: Alianza Editorial, 2020, p. 191.

${ }^{33}$ Ibid., p. 83.

${ }^{34}$ Ibid.
} 
conforme a sus cuerpos, una posición espaciotemporal diferente en el mismo y único espacio total, respectivamente, en el mismo y único tiempo del mundo"35.

En este sentido, podemos decir que la percepción que tiene el yo del mundo es perspectivista. La perspectiva, a propósito, tiene una relación directa con la corporalidad vivida, ya que el mundo se abre en una perspectiva dependiendo del lugar que ocupa el sujeto corporal. Si este sujeto cambia de posición espacial, la perspectiva también cambia. Si dos sujetos intercambian posición, cada uno tiene acceso a lo que era la perspectiva del otro. "Si un cuerpo intercambia su posición espacial con otro se modifican, en una transformación continua, los apareceres que los correspondientes yoes tienen de las cosas de que tienen experiencia, y justamente de tal modo que, en un caso ideal, los apareceres se intercambian a partir del intercambio de los lugares del cuerpo"36.

¿Pero qué ocurre si realmente no me es posible intercambiar el lugar con el otro, si no puedo trasladarme en persona al lugar que ocupa el otro y desde el cual tiene una perspectiva determinada? ¿Ello imposibilita mi comprensión de su lugar o hace imposible que yo tenga experiencia de lo que él ve? Si la respuesta fuese que no, entonces podriamos decir que nuestra experiencia del mundo sería en primer lugar solipsista; en segundo lugar, nuestra representación del mundo se vería empobrecida al depender solo de nuestra experiencia; en tercer lugar, la comunicación, que es un acto social, carecería de sentido -y ya pueden anticiparse las implicaciones de ello para la educación: educar seria imposible-. Hay que concluir, por consiguiente, que hay otros modos de acceder a la perspectiva ajena y tener acceso a las representaciones del mundo ajenas en un sentido amplísimo.

Cabe decir que la empatía es un fenómeno intersubjetivo no en el preciso sentido de que a través de él tengo acceso a las vivencias ajenas, lo que es fundamental también, sino precisamente porque el otro yo también es un sujeto que empatiza ya sea conmigo, ya sea con los demás. Los otros yoes no solo son puestos en empatía, sino que ellos mismos ven, recuerdan y tienen empatías $^{37}$. Por esta razón, Husserl habla de un "contexto intersubjetivo de la conciencia" para referirse a "la experiencia que va de una conciencia de yo a otra conciencia de yo" 38 .

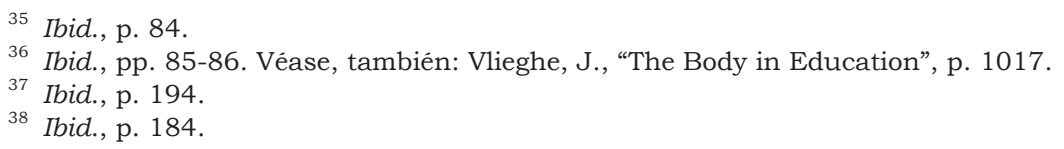


Pues bien, a esta experiencia a partir de la cual podemos aprehender las vivencias ajenas se le llama empatía. Edith Stein trabajó sobre este tema en su tesis doctoral de 1916: Zum Problem der Einfühlung y llama la atención que su obra sigue siendo, por lo menos dentro de las investigaciones fenomenológicas actuales, un punto de referencia esencial y fuente obligada de consulta. En el Capítulo IV de esta obra, Stein habla de "la empatía como comprensión de personas espirituales". De acuerdo con el análisis que presenta, una condición de posibilidad fundamental para la empatía es "la posibilidad del desplazamiento de mi punto cero junto con mi cuerpo físico, y también la posibilidad de un desplazamiento en la fantasía que discrepa entonces del punto cero real y de la orientación que le pertenece" ${ }^{39}$. Gracias al fenómeno de la empatía, Stein puede sostener que el mundo "no se presenta ahora meramente así y después de otra manera, sino de las dos maneras al mismo tiempo. Y se representa distinto no solo dependiendo del respectivo punto de vista, sino también dependiendo de la condición del observador. Con ello, la apariencia del mundo (Welterscheinung) se muestra como dependiente de la conciencia individual, pero el mundo que aparece-que permanece el mismo como quiera y a quien quiera se le aparece-se muestra como independiente de la conciencia" ${ }^{40}$. En efecto, lo que Stein quiere sostener aquí es que la empatía deviene como fundamento de la experiencia intersubjetiva del mundo.

Stein no duda en afirmar que el valor de las representaciones ajenas a las que se tiene acceso mediante la empatía radica, entre otras cosas, en que en ellas hay un enriquecimiento del mundo propio con el mundo ajeno; en la experiencia empatizante se muestra "la posibilidad del enriquecimiento de la propia imagen del mundo a través de la de los otros" y en esto mismo se destaca "la relevancia de la empatía para la experiencia del mundo externo real”" Según esto, puedo acceder a los diversos escorzos del mundo a los que no tengo acceso de manera originaria a través de actos de empatía. En efecto, cada yo se experimenta a sí mismo corporalmente como el punto cero de las coordenadas espaciales; en la empatía, se da el reconocimiento de que mi punto cero aparece en ella como "un punto en el espacio entre muchos". Se da entonces mi cuerpo entre otros cuerpos, mi perspectiva dentro de muchas otras perspectivas y mis campos de sensación como una posibilidad de muchos otros campos de sensación.

${ }^{39}$ Edith Stein, Zum Problem der Einfühlung, p. 81.

${ }^{40}$ Ibid., p. 81ss.

${ }^{41}$ Ibid., p. 80. 
Por estas razones, nos parece apropiado decir que nuestra experiencia intersubjetiva del mundo es una experiencia intercorpórea. Nos relacionamos con los otros a través del cuerpo y de la noticia corporal que tenemos de ellos y ellos de nosotros. En efecto, el cuerpo del otro me es dado como cuerpo vivo que es portador de libre movimiento y este movimiento no es un movimiento mecánico, sino el movimiento de un cuerpo vivo. En este sentido, Vargas Guillén lleva razón al decir que la empatía tiene el carácter de base de la intersubjetividad, esto es, de la relación de los-unos-con-los-otros ${ }^{42}$.

Pues bien, Ingrid Vendrell ha señalado con razón que el énfasis de Edith Stein sobre el cuerpo vivo para el análisis de la intersubjetividad en contraste con el de otros fenomenólogos, como es el caso de Scheler, es crucial para el conocimiento de otras mentes ${ }^{43}$, o sea, para comprender la aprehensión de las vivencias ajenas. Gracias a que empatizamos con las sensaciones corporales de otros individuos, señala Vendrell, es que nos es dada una multiplicidad de perspectivas o puntos de vista sobre el mundo. Pero, más allá de ello, uno de los aspectos importantes de la empatía en relación con el cuerpo es el fenómeno de la expresión ${ }^{44}$.

El cuerpo vivo propio y el ajeno expresan. ¿Qué es lo que expresan? El cuerpo vivo es expresión del espíritu. En él se expone la vida interior de la persona. "La sonrisa en la que mi alegría se exterioriza según la vivencia me está dada, a la vez, como una distorsión de mis labios"45, de la cual se tiene una percepción externa. No obstante, cabe decir que "fenómenos de expresión distintos se muestran como fenómenos de percepción iguales. Enrojezco de cólera, de vergüenza y de esfuerzo; en todos los casos tengo la misma percepción de que 'me sube la sangre a la cara'. Pero una vez vivencio dicho proceso como expresión de la cólera, otra como expresión de la vergüenza y otra de ningún modo como expresión, sino como consecuencia causal del esfuerzo"46. Sin embargo, la modificación corporal no es suficiente para comprender el fenómeno de la expresión. "Fruncir el ceño de enfado y fruncir el ceño para simular enfado son claramente diferenciables en sí, incluso cuando paso de la percepción corporal a la percepción externa"47.

\footnotetext{
${ }^{42}$ Vargas, G., Fenomenología y performance, Bogotá: Aula de Humanidades, 2019, p. 76.

${ }^{43}$ Vendrell, I., "Empathy, Emotional Sharing and Feelings in Stein's Early Work", en: Human Studies, v. XXXVIII, 2015, pp. 481-502.

44 Ibid., p. 499.

45 Stein, E., Zum Problem der Einfühlung, p. 71.

46 Ibid.

${ }^{47}$ Ibid.
} 
Pero la tesis que es sumamente relevante para la empatía es la que dice que estos fenómenos "que aparecen como afluencia de los sentimientos son, a la vez, expresión de las propiedades anímicas que en ellos se manifiestan: la mirada rabiosa delata un temperamento fuerte". ${ }^{48}$ Esto apunta, entonces, a que mediante el fenómeno de la expresión tengo un conocimiento del carácter de la persona. En la manera como reacciona, como responde a lo que le pasa, conozco una parte de sus propiedades personales como el carácter o el temperamento. En efecto, lo que Stein quiere mostrar es la importancia de las vivencias de sentimiento para la constitución de la personalidad propia y para el conocimiento de la personalidad ajena, lo que resulta de sumo valor para el fenómeno de la educación. Dado que los sentimientos tienen cierta profundidad, en la experiencia que tiene de ellos la persona, tiene una experiencia de sí mismo y por ello las experiencias afectivas le descubren a la persona sus propios estratos personales, el modo como afectivamente está dispuesto en el mundo. Mediante las experiencias afectivas, esto es, mediante los sentimientos y las emociones, la persona descubre los distintos campos axiológicos, esto es, los valores, a partir de los cuales puede encontrar las motivaciones para actuar, para ponerse en movimiento y decidirse por algo. Los valores aparecen "como diversas luminarias de cuya posición y fuerza lumínica depende la iluminación resultante" 49 . Además, la profundidad desde la cual se vive el sentimiento, a juzgar de Stein, es "correlativa a la altura del valor sentido" y de ello depende también "el 'radio de acción' del estado de ánimo suscitado"50.

$\mathrm{El}$ conocimiento y contacto con otras personas resulta de vital importancia una vez más. En primer lugar: porque hay cierto tipo de sentimientos que solo se hacen posibles a través del contacto con otras personas, tales como el amor, el odio, la gratitud, el rencor, la envidia o la venganza, ya que son "sentimientos que tienen por objeto a otras personas" 51 y en cuyo vivenciar se descubren los estratos o niveles de profundidad de nuestra persona. Stein muestra que el contacto con otras personas es importante, especialmente desde el punto de vista de la empatía, porque a través de las vivencias ajenas tenemos acceso a valores que originalmente no me serian dados en mi propia persona. No quiere decir esto que no pueda apropiarme de esos valores y descubrirlos en mí, sino que los descubro en otro u otros y al mismo tiempo reconozco que esos valores

\footnotetext{
48 Ibid., pp. 71 ss.

49 Ibid., p. 122.

50 Ibid.

51 Ibid., p. 119.
} 
no me pertenecen, no son míos. Stein habla, así, de "dominios axiológicos" que originalmente están "clausurados para nosotros" y a los cuales tenemos acceso gracias al contacto interpersonal; asi "con los nuevos valores obtenidos en empatía se abre simultáneamente la mirada a valores desconocidos en la persona propia"52.

Por consiguiente, la empatía hace posible un enriquecimiento del mundo al tener acceso a una pluralidad de perspectivas que pertenecen a otros; $y$ hace posible también el conocimiento de dominios axiológicos ajenos que pueden ayudarnos a conocer nuestras propias carencias axiológicas o bien contribuir a la puesta en movimiento de la existencia a fin de ganar para nosotros esos dominios axiológicos que son de otros y sirven de motivación personal. En este segundo caso, la persona puede verse motivada por los otros a fin de crecer espiritualmente y apropiarse poco a poco de algo que carece y el otro le muestra. Las consecuencias que la empatía tiene para la educación empiezan a perfilarse en este punto.

\section{Empatia y educación}

La empatía la hemos entendido, siguiendo a Edith Stein, como "la aprehensión de vivencias ajenas". En los apartados precedentes, hemos explorado las relaciones entre la intersubjetividad y la educación y entre la empatía y la intersubjetividad. Con ello, hemos sentado las bases para poder afirmar que la vida humana es esencialmente intersubjetiva y que la experiencia o vivencia del cuerpo vivo propio y ajeno ocupan un lugar esencial. Así, una de las dimensiones o fenómenos que se presentan en la vida humana es el que llamamos educación. Lo que vamos a sostener en este apartado es, entonces, que la empatía está en la base del fenómeno de la educación y, por tanto, que los presupuestos o condiciones que se presentan en los actos empatizantes se dan también en los procesos de educación. Más aun: queremos argumentar que la empatía es necesaria para la educación, como es necesaria en los modos como los sujetos se relacionan unos con otros.

De acuerdo con V. Costa, como hemos visto supra, en el fenómeno de la educación un sujeto abre a otro a las propias posibilidades. Ahora debemos cuestionar: ¿qué significa esta apertura? A juicio de Stein, esta apertura es una nota esencial de la persona humana, esto es, su posibilidad de abrirse y, en consecuencia, la posibilidad también de cerrarse. La persona, además, se

52 Ibid., p. 135. 
constituye en una doble apertura, a saber, hacia dentro y hacia fuera ${ }^{53}$. A partir de ello, puede conocerse a sí misma y puede conocer el mundo que le rodea, entrar en contacto con otras personas. De la misma manera, parece razonable sostener que la persona puede cerrarse al conocimiento de sí misma y al conocimiento del mundo externo ¿Qué importancia tiene ello para la educación y de qué modo la empatía se cumple? Hay experiencias traumáticas que pueden tener como consecuencia que la persona se cierre, para aprender, por ejemplo, si tiene una experiencia desafortunada en la escuela, o que tenga dificultad para socializar con otras personas si la han dañado en el pasado, etcétera.

Sin embargo, ¿cómo es posible que las vivencias de unos individuos estén al alcance de otros y puedan ser aprehendidas? La respuesta está en la empatía: en la posibilidad de que los individuos participen de los recuerdos, las expectativas, los campos de percepción, la experiencia corporal unos de otros y, además, que "se abran unos a otros y asuman una actitud de comprensión recíproca" 54 . Esta "comprensión recíproca" se da en la intersubjetividad mediante la comunicación mutua.

La educación formal, esto es, la educación en la escuela, como ha señalado Kiymet Selvi, tiende a ser uniforme, partiendo de que las personas tienen sentimientos, conocimientos y comportamientos similares, pero pasan por alto las diferencias individuales de los estudiantes ${ }^{55}$. Las diferencias individuales, al ser notas constitutivas de la persona, son necesarias. Puesto que se trata de abrirse unos a otros y buscar una comprensión recíproca, lo que se busca en la educación, ya sea en la escuela, ya sea en la familia o en cualquier plano de la vida, consistiría en animar o motivar a las personas a que compartan sus experiencias. Desde una perspectiva fenomenológica, debe buscarse el significado, es decir, el sentido de las experiencias vividas. Por ello, los sujetos de la educación pueden describir sus experiencias, abrirse unos a otros, considerar ellos mismos y con ayuda de los otros los posibles significados de sus vivencias, como sus dificultades para el aprendizaje, en las situaciones personales en las que viven en su comunidad, en su familia, sus aspiraciones, deseos, sueños, el proyecto de vida o vocación, etcétera. Comprender al sujeto de la educación implica comprenderlo como persona y, en consecuencia, comprender el horizonte de sentido en el que se despliega su vida.

\footnotetext{
${ }^{53}$ Stein, E., Der Aufbau der menschlichen Person. Vorlesung zur philosophischen.

${ }^{54}$ Stein, E., Zum Problem der Einfühlung, p. 125.

${ }^{55}$ Selvi, K., "Phenomenological Approach in Education", en: Analecta Husserliana, v. XCV (2008), p. 39.
} 
Como indica Selvi ${ }^{56}$, las vivencias de los estudiantes incluyen sentimientos, pensamientos y respuestas, así como la relación que tienen con su sí mismo y con los otros, tanto estudiantes como maestros, familiares, vecinos, entre otros. Los sujetos de la educación están en contacto con otros, viven en un mundo con facilidades y dificultades que tienen que enfrentar, con una vocación que les llama a ser de un modo determinado. En este sentido, la pedagogía fenomenológica "apoya a los profesores y estudiantes en aprender y enseñar haciendo hincapié en sus individualidades únicas" ${ }^{\text {77 }}$. Esto es así debido a que las personas, en palabras de Husserl, “...son, por principio, unidades de desarrollo infinito... las personas devienen y son objetos para realizaciones personales" 58 .

Selvi, en efecto, enfatiza en lo importante que es la fenomenología para la educación desde el punto de vista desde el cual cada persona (cada estudiante, cada profesor) tiene experiencias propias, se enfrenta a situaciones distintas, aprende de modos diferentes, experimenta, siente, desea y demás. Las actividades de aprendizaje de las que habla allí, están centradas en el conocimiento, las opiniones, los valores, la percepción, la libertad y la comprensión desde la primera persona. Este nos parece un punto de partida importante, pero no nos dice nada o nos dice muy poco de lo que ocurre con los otros y lo importante que son esas experiencias cuando las percibimos en los otros, o las imaginamos, recordamos, fantaseamos, etcétera. No se dice nada de la empatía, y esta es necesaria cuando hablamos de un proceso que se vive de manera intersubjetiva. Justamente porque en este encuentro interpersonal unos sujetos se ven influenciados por otros, porque en las relaciones interpersonales se vive la empatía de muchas formas, tales como la simpatía, la apatía o la antipatía y de despiertan sentimientos cuyo punto de llegada son los otros: el amor, el odio, la envidia, el cariño, la venganza, entre otros tantos más.

Por su parte, Brinkmman y Friesen sostienen que esa pluralidad de significados que se dan en la experiencia de los sujetos "surgen de la posición, el interés y el contexto de cada uno y de acuerdo con las estructuras espaciotemporales, intersubjetivas e (in)materiales". ${ }^{59}$ Subrayan que cada experiencia "se sitúa en un horizonte que abarca experiencias, recuerdos y esquemas

\footnotetext{
56 Ibid., p. 39.

57 Ibid., p. 42.

${ }^{58}$ Husserl, E., "El espíritu común (Gemeingeist) I y II (1921)”, en: Thémata, 1987, p. 154.

59 Brinkmman, M. y N. Friesen, "Phenomenology and Education", en: Smeyers, P. (ed.), International Handbook of Philosophy of Education, v. I, Nueva York: Springer, 2018, p. 591.
} 
anteriores y también anticipaciones y expectativas, así como su realización o decepción"60.

La empatía hace posible que el mundo de unos y otros se enriquezca, ya que, gracias a que tenemos acceso a las vivencias ajenas, nuestra percepción del mundo es mayor, los valores a los que tenemos acceso crecen, la representación de distintas formas de vida y estilos personales hace posible una mayor tolerancia y una mejor convivencia, así como la falta de comprensión conduce en muchos casos al rechazo, la exclusión y la violencia. El paso del mundo familiar al mundo extraño no parece ser posible sin la capacidad del reconocimiento del otro, tanto de su persona como de su cultura, su estilo, por ende, sus tradiciones y estilos de vida. La empatía nos abre a nuevas posibilidades de apropiación y de trato con los otros. Las experiencias ajenas son fuente de la cual emanan nuevos sentidos y nuevos horizontes de acción, ya que en la empatía la persona sale al encuentro de lo desconocido, de lo que originariamente es de otro. "Este mundo es un mundo, y es uno y el mismo para cada (yo) que experiencia, el cual en este mundo como mundo circundante de un yo que primero se presupone, es objeto de experiencia, y que pertenece a él en carne y hueso, y es seguido y comprendido espiritualmente por la experiencia de la empatia"61.

La educación es un fenómeno intersubjetivo que requiere de la comunicación de unos con otros. Ello hace posible la vida en común, la comunidad o común unidad de vida. "Más bien estamos en comunicación mediante actos sociales [-sostiene Husserl-]. No vivimos solo uno junto al otro, sino uno en el otro. Nos determinamos el uno al otro de persona a persona, de yo a yo, y nuestra voluntad no va solo a los otros como cosas del mundo circundante, sino que entra en los otros, se prolonga hasta el interior del otro, que es el querer del otro y al mismo tiempo nuestro querer, de modo que un acto suyo, si bien modificado en diversos modos, puede convertirse en un acto nuestro"62.

Quienes se educan juntos comparten su vida con los otros, comparten mundos de intereses, planes de vida, proyectos y metas en común, conocimientos, estados de ánimo, experiencias en general. Se trabaja persiguiendo los mismos fines, los mismos objetivos, pero además este vivir es un con-vivir, un vivir juntos, un llevar unos a otros y el dejarse motivar y afectar unos por otros. "Vivir en comunidad significa compartir con otros, estar en contacto

\footnotetext{
60 Ibid., p. 592.

61 Vargas, G., Fenomenología y performance, p. 80.

62 Husserl, E., "Valor de la vida, valor del mundo. Moralidad, virtud y felicidad", en: Acta fenomenológica latinoamericana, v. III (2009), p. 805.
} 
con ellos a través de formas de pensar, sentir, querer y actuar juntos. Vivir en común e inclusive dejarse influenciar por ellos, sentir con ellos y a través de ellos -como ocurre a través de la empatía-". ${ }^{63}$ Como decía Husserl, no vivimos nuestra vida de manera solipsista, sino que nos dejamos motivar y determinar unos a otros. No vivimos unos junto a otros, sino unos en los otros. El querer de los otros se convierte en el querer nuestro. Los deseos y aspiraciones mías llegan a ser deseos y aspiraciones también de los demás. "Mi pensamiento abarca otro pensamiento -tanto propio como ajeno- como acto activamente eficiente. Mi querer abarca el querer ajeno y mi acción incluye en sí la acción ajena (o bien será abarcada por ella)"64.

Antoniou y Karavakou argumentan que la educación actual debe recuperar el sentido de la paideiay, a partir de alli, debe centrarse en los "esfuerzos autoformativos, autotransformadores y autorealizantes de un sujeto capaz y consciente" 65 . Argumentan, en efecto, que la subjetividad no representa un problema metodológico, "ya que las narrativas personales son fuentes auténticas de cómo lidiamos con situaciones de la vida real"66. En concordancia, entonces, con nuestra argumentación y nuestro punto de vista, Antoniou y Karavakou sostienen que, desde la subjetividad o experiencia vivida, los sujetos validan la objetividad del conocimiento y "colocamos cúmulos narrativos subjetivos de significado acumulado en una relación dialogada con la cultura"67. Ciertamente, quien investiga o imparte clases no está literalmente en el punto de vista del otro, por ejemplo, del estudiante, pero se requiere (como en el caso de la investigación educativa) que la narración de los participantes sea tomada en serio. Es lo que sostienen Antoniou y Karavakou y es lo que sostenemos nosotros desde un enfoque basado en la empatía.

En todas las experiencias los sujetos dan sentido a lo vivido, en la experiencia se atribuye significado a las cosas, pero cabe decir que, desde un punto de vista fenomenológico, el modo como las cosas llegan a tener sentido no está determinado, sino que está en juego un horizonte vital más amplio como pueden ser los motivos y los intereses de la persona, la historia personal y, por qué no, factores determinantes como la edad, el sexo, la posición social y el medio en que se habita, la relación que se tiene con los valores culturales, la ciencia, la

\footnotetext{
${ }^{63}$ Sánchez, R., Persona y afectividad. Invitación a la fenomenología de Edith Stein, Bogotá: Aula de Humanidades, 2020, p. 81.

${ }^{64}$ Husserl, E., "El espíritu común”, p. 152.

65 Antoniou, K. y V. Karavakou, "Education in Crisis”, p. 209.

66 Ibid., p. 210

67 Ibid., p. 210.
} 
tecnología, la religión, en fin: el universo personal desde el cual la experiencia llega a significar una cosa u otra. Al margen de todo ello, ¿se puede hablar de vivencias sin sentido? No parece razonable hablar de sinsentido o falta de sentido por una razón: porque el sentido es sentido vivido y lo que no es vivido en primera persona es vivido por otros. Alli es donde aparece la empatía como fenómeno que nos da acceso a universos de sentido que originariamente son de otros y donde cobra validez el diálogo, la escucha y el encuentro. En este caso, lo que importa, como dice Waldenfels, es "que algo se muestra como algo" y que precisamente "aparece así y no de otra manera"68. Eso no quiere decir que no resulte problemático que el sentido llegue a depender de una determinada perspectiva o que un sentido sea preferido por encima de otros posibles dependiendo del contexto, como problemático resulta también sostener que existe el sentido, pero no un único sentido ${ }^{69}$. En otro plano, esto es equiparable a decir que existe la cultura, pero no una única cultura.

Este acto de dar sentido o significado, esto es, la constitución de un objeto en la experiencia vivida, ¿'es solipsista? ¿'Se trata de un fenómeno inmanente? La fenomenología ha puesto especial atención a la experiencia vivida en primera persona, pero que esto sea así no debe entenderse en el sentido de que la vida subjetiva es solipsista, puesto que, como hemos visto, nuestra experiencia del mundo es intersubjetiva. La experiencia escolar o cualquier relación que suponga la educación de un sujeto personal se da a través de actos sociales, del encuentro de unos con otros. En las relaciones entre maestros y estudiantes, como observan Antoniou y Karavakou, aparece la influencia de varios factores, tales como: "a) el lugar (el aula); b) el tiempo (por ejemplo, la edad de uno, las experiencias previas, el ocio); c) las expresiones corporales; y d) los inefables significados subjetivos" 70 . A juicio de Henriksson, debe cultivarse "una relación especial entre profesores y estudiantes" y promoverse entre ellos un "encuentro de miradas". Ello sería propio de una "auténtica pedagogía" 71.

Nuestro juicio al respecto es, entonces, que este "encuentro de miradas" y esta "relación especial" entre los sujetos involucrados en los procesos de educación, está mediada por la empatía, la cual es un fenómeno que, al igual que la educación, aparece entre nosotros. En este caso, la comunicación o actos

\footnotetext{
${ }^{68}$ Waldenfels, B., Exploraciones fenomenológicas acerca de lo extraño, pp. 19 y 21. Énfasis del autor.

${ }^{69}$ Ibid., p. 21.

${ }^{70}$ Antoniou, K. y V. Karavakou, "Education in Crisis", p. 219.

${ }^{71}$ Ibid.
} 
sociales son de sumo valor: el diálogo, la escucha, el encuentro personal, el abrirse unos a otros para compartir, convivir, trabajar, entre otros. La empatía hace posible el enriquecimiento de la propia vida a partir de la vida de los otros: este es el punto de partida de la educación, bajo el entendido de que ella supone el encuentro de dos o más sujetos. Para la fenomenología, la subjetividad constituye el punto de partida de la investigación educativa. "La fenomenología ofrece información sustancial sobre lo que realmente sucede con el individuo y las narraciones personales constituyen excelentes fuentes de conocimiento sobre la forma en que la subjetividad se ocupa de la vida real"72.

¿Por qué la fenomenología es importante, entonces, para el estudio del fenómeno educativo? Porque muestra que los sujetos involucrados en la educación están intersubjetivamente relacionados. Esta intersubjetividad necesita y presupone la empatía, porque en ella se da un reconocimiento mutuo de los sujetos involucrados. Además, entre ellos hay una constante retroalimentación: en la escuela, por ejemplo, el alumno aprende del maestro, se deja guiar, se deja llevar y conducir, pero también el maestro retroalimenta su trabajo, sus métodos, sus técnicas, en función de lo que observa con sus alumnos. Es la experiencia vivida en este encuentro interpersonal el que hace posible que los sujetos de la educación se pongan en movimiento y se transfieran sentimientos, emociones, ideas, conceptos, costumbres, estilos de vida, valores, hábitos, virtudes y prácticas, etcétera ${ }^{73}$. Ahora bien, este énfasis en la intersubjetividad no es propia ni exclusiva de la fenomenologia -la encontramos también en la teoría crítica o en la hermenéutica-, pero es el enfoque que nos parece más apropiado para describir el fenómeno de la educación.

Por ello, la subjetividad de la que se habla aquí no es la subjetividad individual, aislada a la manera del solipsismo, sino de una subjetividad que debe ser entendida como intersubjetividad: "los sujetos humanos se constituyen en y a través de las prácticas culturales educativas mientras que su autoconstitución es responsable de la constitución del espacio cultural"74. El sujeto de la educación tiene la posibilidad de volver la mirada sobre su propia experiencia vivida. "Solo en la experiencia, en su vuelta sobre sí misma, que al mismo tiempo es un cambio en nuestra capacidad de experimentar, reside el verdadero poder educativo de la experiencia" -sostiene Buck-75.

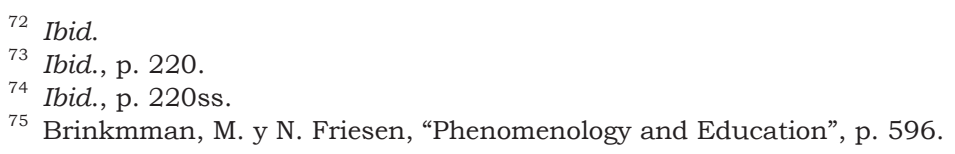


Werner Loch, como recuerdan Brinkmman y Friesen, habla del aprendizaje y el curso de la vida tomando en cuenta el importante lugar que ocupa el cuerpo para el aprendizaje. "El cuerpo vivido adquiere importancia como categoría de reflexión y como fenómeno en sí mismo, y el aprendizaje está relacionado con los hábitos sedimentados y el hábito en general"76. El cuerpo vivo o propio (Leib) es importante en toda actividad o proceso educativo, ya que nos valemos del cuerpo para actuar, para crear, para expresar. El aprendizaje situado lo es no solo por tener en consideración el mundo entorno del sujeto de la educación, sino que hace referencia de modo implícito a la relación que tiene la perspectiva con el cuerpo, con el modo como habitamos corporalmente en el espacio -usando una expresión de B. Waldenfels-, mas no todos los sujetos habitamos de la misma manera. Están en juego una multiplicidad de factores.

El mundo se abre para nosotros en una perspectiva concreta que puede ampliarse en una pluralidad de perspectivas fantaseadas. Mi cuerpo vivo ocupa el punto cero de las coordenadas espaciales y en la experiencia de cuerpos vivos ajenos se nos dan otros puntos ceros de coordenadas, pero en la modalidad de no ser propios, sino ajenos. Esas otras perspectivas son en algunos casos producto de la imaginación y la fantasía, y en muchos otros casos son resultado de la empatía: son perspectivas del mundo ajenas, que pertenecen originariamente a otros. "A partir de mi orientación corporal hacia el mundo y los demás [-señala Orlikovski-], el cuerpo extraño se reproduce desde mi cuerpo a través de la modalidad de una 'transmisión aperceptiva'. El otro se presenta solo 'indirectamente' en el sentido de subjetividad soberana. El hecho de que el cuerpo percibido contenga una dimensión psíquica es esencialmente posible gracias a la modalidad de 'empatía"'77.

El cuerpo propio ocupa un lugar importante en y para la educación, ya que, aunque la mayor parte del tiempo es olvidado, siempre está presente. El cuerpo propio está allí donde está la persona y es un punto de referencia esencial para el trato con ella. Por esta razón, como señala Vlieghe, "la corporalidad se considera un factor importante a tener en cuenta al teorizar en educación"78. El cuerpo, dice, ha sido redescubierto por los filósofos de la educación, aunque en la práctica diaria de la enseñanza y el aprendizaje permanezca en el olvido.

\footnotetext{
${ }^{76}$ Ibid.

${ }^{77}$ Orlikovski, A., "Das Subjekt des Lernens", en: Brinkmann, M. y otros (eds.), Pädagogick-Phänomenologie. Verhältnisbestimmungen und Herausforderungen, v. III, Gewerbesrasse: Springer Nature, 2017, p. 146

${ }^{78}$ Vlieghe, J., "The Body in Education", p. 1013
} 
Algunos fenomenólogos, entre ellos Edmund Husserl, Edith Stein, Maurice Merleau-Ponty, entre otros, trabajaron en la descripción de la vivencia corporal. Estas descripciones pueden llevarse al campo propio de reflexión en materia de educación. ¿Cómo llega a tener sentido el mundo de la educación para la persona dependiendo de su edad, de su sexualidad, de sus condiciones físicas desde la normalidad o anormalidad, en la salud o en la enfermedad?79

La normalidad y la anormalidad, así como lo familiar y lo extraño, ocupan un lugar importante dentro de las investigaciones fenomenológicas y son, a nuestro juicio, elementos clave para comprender el fenómeno de la educación. Aquí no es posible desarrollar las diferencias entre ellos, pero es posible decir que se hallan indicaciones importantes en la obra de Husserl y, además, ha sido B. Waldenfels quien mayormente se ha dedicado a la descripción del fenómeno de lo extraño. Lo que pasa es que ni lo familiar o normal pueden comprenderse sin sus opuestos: lo extraño y anormal. También es verdad que se trata de términos que pueden relativizarse, esto es: lo familiar y normal para unos es extraño y anormal para otros. Al relativizarse, lo que decimos es que el mundo toma un sentido u otro dependiendo del modo como se vive o constituye por los sujetos, de cómo se está o habita el mundo ${ }^{80}$. Por esta razón, Rosemary Rizo-Patrón se refiere al "mundo de la vida subjetivo-relativo" que se constituye desde la dimensión de la intersubjetividad ${ }^{81}$. Lo importante, para el caso que nos ocupa, es que ya dentro de nuestro mundo circundante conocido, es decir, dentro de nuestro mundo familiar, aparecen ya la otredad, la diferencia o, en otras palabras, lo extraño. Tanto lo familiar (lo más conocido, presente y próximo) como lo extraño (lo menos conocido, lo ausente y lejano) aparecen y se dan dentro del horizonte de la intersubjetividad ${ }^{82}$. De este modo, nos parece razonable sostener que lo extraño, en tanto que se refiere a lo otro, apunta ya de manera originaria a lo diferente, a lo que pertenece a otro y es distinto (distinto, claro, de lo propio).

Por ello, a juicio de Rizo-Patrón, los términos "lo mismo" y "lo otro", así como "identidad y diferencia", resultan ser "dialécticamente interdependientes" y nos sitúan "en el terreno de la pluralidad". Esta pluralidad aparece ya en el mundo de la vida en la forma de "la pluralidad de mónadas; de comunidades

\footnotetext{
${ }^{79}$ Carel, H., Phenomenology of Illness, Oxford: Oxford University Press, 2016.

${ }^{80}$ Para profundizar en este tema puede verse: Husserl, E., Meditaciones cartesianas, México: Fondo de Cultura Económica, 2005, especialmente la "Quinta meditación"; Waldenfels, B., Exploraciones fenomenológicas acerca de lo extraño; Rizo-Patrón, R., El exilio del sujeto, p. 313ss.

${ }^{81}$ Cf. Rizo-Patrón, R., El exilio del sujeto.

82 Ibid., p. 303.
} 
de comunicación y de "personalidades de orden superior" -como la pluralidad de naciones y correativamente de "mundos familiares", de ethoiy de culturas-; en fin, incluso la pluralidad de epistemes" 3 .

Pues bien, la forma en como el mundo se nos presenta está mediada por nuestra corporalidad, la cual está inserta en el mundo ${ }^{84}$. ¿Qué diferencias hay en la constitución de una subjetividad en su edad adulta a diferencia de una edad infantil o en la vejez? ¿Qué ocurre en los casos de enfermedad o en los que el sujeto ha sufrido lesiones en su cuerpo o ha perdido una extremidad? ¿De qué modo se constituye el mundo para quien ha perdido la vista o el oído? Aunque no de un modo exclusivo, aquí es donde la fenomenología puede hacer aportes significativos a la educación inclusiva ${ }^{85}$.

El vivir unos con otros en los procesos de educación, abre el horizonte, además, de una serie de problemas que apuntan a los modos distintos que tenemos de relacionarnos con el mundo dependiendo de la generación a la que pertenecemos. El horizonte intersubjetivo de la educación exhibe ahora otra dimensión importante de la misma: el vivir unos con otros de manera intergeneracional. En el mismo espacio y en el mismo tiempo, conviven distintas generaciones, están en juego distintos sistemas de creencias, se oponen estilos de vida, modos de ver el mundo y actuar en él, distintos sistemas de valoraciones, hábitos diferentes, etcétera. El Capítulo 1 de su libro Fenomenología continuada, lo dedica Lester Embree a describir "diferencias generacionales" 86 . Estas diferencias, cabe decir, son importantes para comprender el fenómeno de la educación y para aproximarnos a la comprensión de la empatía en un mundo intersubjetivo, intercorpóreo e intergeneracional. Por las mismas razones, se trata de un mundo intercultural: el sujeto de la educación vive entre culturas, entre un mundo familiar y muchos mundos extraños a los que se va aproximando, tratando de comprender su sentido.

La educación nos permite superar esas "incomprensibilidades iniciales" permitiéndonos entrar en lo extraño desde lo familiar, yendo de lo más próximo a lo más lejano. En este sentido, todo proceso de educación supone cierto esfuerzo, justo porque se trata de aprender algo nuevo, o sea algo que originariamente es desconocido. Desde una perspectiva corporal, el aprender

\footnotetext{
${ }^{83}$ Ibid., p. 313ss.

${ }^{84}$ Vlieghe, J., "The Body in Education", p. 1018.

${ }^{85}$ Véase nuestro trabajo en Sánchez, R. y R. Casales, "Empatía, reconocimiento e inclusión", en: Devenires, v. XXII, 44 (2021).

${ }^{86}$ Embree, L., Fenomenología continuada. Contribución al análisis reflexivo de la cultura, Morelia: Jitanjáfora, 2007.
} 
a leer supone el movimiento del cuerpo, el estar en una posición, el enfocar la mirada en un ángulo y una distancia determinada. Además, el escribir supone lo anterior y también el movimiento de la mano, el poder manipular con la mano un objeto (un lápiz, un boligrafo, una pluma, etcétera), el llevarlo de un lado a otro realizando ciertos trazos, ejerciendo cierta presión, trazos determinados. Los ejercicios de caligrafia dependen de los movimientos de la mano y de la posibilidad de lograr cierta familiaridad con el objeto tanto material (como lo es el lápiz) como inmaterial (como son las letras y las palabras). En realidad, no se trata solo de la mano: en toda actividad de aprendizaje está en juego el cuerpo entero, la persona entera, tanto en sus cualidades fisicas como en sus facultades intelectivas. ¿Por qué no tendría que tomarse en cuenta el cuerpo si es la dimensión personal que nos acompaña y hace posible, junto con muchos otros elementos, el aprendizaje?

\section{Conclusiones}

En este trabajo hemos descrito desde un enfoque fenomenológico la importancia que tiene la empatía para la educación, y lo hemos realizado recuperando la dimension intersubjetiva de la persona. En esta intersubjetividad, la empatía hace posible la comprensión de las vivencias psíquicas ajenas. Para ello, como hemos visto, el cuerpo ocupa un lugar fundamental. La intersubjetividad devine intercorporalidad, relaciones subjetivas mediadas por la experiencia corporal, por el habitar corporalmente en el mundo y, en consecuencia, en la escuela. Toda actividad de enseñanza-aprendizaje, toda actividad de educación, se vale del cuerpo para crear, para comunicar, para llegar a hacer algo. Pero hemos visto que toda educación se da en el horizonte de la cultura, en un ir de lo familiar a lo extraño, y en este sentido, la intersubjetividad deviene interculturalidad; el estar unos con otros conviviendo entre las culturas, viviendo en el esfuerzo de comprendernos unos a otros. Esta comprensión se hace posible a través de la empatía y la empatía, en consecuencia, es el fundamento de la educación. Una pregunta que dejamos sin formular y sin responder: ¿se puede enseñar y, correlativamente, se puede aprender la empatía? Dejaremos esta pregunta para otro trabajo.

Recibido: 08/12/2020

Aceptado: 07/06/2021 


\section{Bibliografia}

Altarejos, F. y C. Naval, Filosofía de la educación, Pamplona: EUNSA, 2011.

Antoniou, K. y V. Karavakou, "Education in Crisis: Reflections on the Contribution of Phenomenology to Modern Educational and Political Culture", en: Brinkmann, M. y otros (eds.), Pädagogik-Phänomenologie. Verhältnisbestimmungen und Herausforderungen, v. III, Gewerbesrasse: Springer Nature, 2017, pp. 209-223. https://doi.org/10.1007/978-3-658-15743-2_12

Barreiro, J., "Educación y concienciación”, en: Freire, P. (ed.), La educación como práctica de la libertad, México D.F.: Siglo XXI, 2020.

Brinkmman, M. y N. Friesen, "Phenomenology and Education", en: Paul Smeyers (ed.), International Handbook of Philosophy of Education, v. I, Nueva York: Springer, 2018, pp. 591-608. https://doi.org/10.1007/978-3-319-72761-5_46

Carel, H., Phenomenology of Illness, Oxford: Oxford University Press, 2016. https://doi. org/10.1093/acprof:oso/9780199669653.001.0001

Costa, V., Fenomenología de la educación y la formación, Salamanca: Sígueme, 2018.

Embree, L., Fenomenología continuada. Contribución al análisis reflexivo de la cultura, Morelia: Jitanjáfora, 2007.

Gallagher, S. y D. Zahavi, Phenomenological Mind, Londres: Routledge, 2020. https:// doi.org/10.4324/9780429319792

Husserl, E., "El espíritu común (Gemeingeist) I y II (1921)", en: Thémata, 1987, pp. 131-158.

Husserl, E., Renovación del hombre y de la cultura. Cinco ensayos. Barcelona/México: Anthropos/UAM, 2002.

Husserl, E., Meditaciones cartesianas, México D.F.: Fondo de Cultura Económica, 2005.

Husserl, E., "Valor de la vida, valor del mundo. Moralidad, virtud y felicidad", en: Acta fenomenológica latinoamericana, v. III (2009), pp. 789-821.

Husserl, E., Problemas fundamentales de la fenomenología, Madrid: Alianza Editorial, 2020.

Iribarne, J., En torno al sentido de la vida. Ensayos fenomenológicos sobre la existencia, México: Jitanjáfora, 2012.

Levinas, E., Ética e infinito, Madrid: Machado Libros, 1991.

Magrì, E., "Subjectivity and Empathy: A Steinian Aproach", en: Mariani, E., Discipline Filosofiche: Figures, Functions and Critique of Subjectivity beginning from Husserlian Phenomenology, XXV, 2 (2015), pp. 129-148.

364 Paidós, 2012. https://doi.org/10.2307/j.ctvt7x63g

Orlikovski, A., "Das Subjekt des Lernens", en: Brinkmann, M. y otros (eds.), PädagogickPhänomenologie. Verhältnisbestimmungen und Herausforderungen, v. III, Gewerbesrasse: Springer Nature, 2017, pp. 143-156. https://doi.org/10.1007/ 978-3-658-15743-2_8

Ortega y Gasset, J., “Ensimismamiento y alteración”, en: Obras completas, v. V, Madrid: Taurus/Fundación José Ortega y Gasset-Gregorio Marañón, pp. 529-550. 
Rizo-Patrón, R., El exilio del sujeto. Mitos modernos y posmodernos, Lima: Aula de Humanidades/Fondo Editorial de la Pontificia Universidad Católica del Perú, 2014.

Sánchez, R., Persona y afectividad. Invitación a la fenomenología de Edith Stein, Bogotá: Aula de Humanidades, 2020.

Selvi, K., "Phenomenological Approach in Education", en: Analecta Husserliana, v. XCV (2008), pp. 39-51. https://doi.org/10.1007/978-1-4020-6302-2_4

Stein, E., Der Aufbau der menschlichen Person. Vorlesung zur philosophischen Antropologie, Friburgo: Herder, 2004.

Stein, E., Zum Problem der Einfühlung, EsGA 5, Friburgo: Herder, 2008.

Stein, E., Una investigación sobre el Estado, Madrid: Trotta, 2019.

Vargas, G., Fenomenología y performance, Bogotá: Aula de Humanidades, 2019.

Vendrell, I., "Empathy, Emotional Sharing and Feelings in Stein's Early Work", en: Human Studies, v. XXXVIII (2015), pp. 481-502. https://doi.org/10.1007/ s10746-015-9346-4

Vlieghe, J., "The Body in Education", en: Smeyers, P. (ed.), International Handbook of Philosophy of Education, v. II, Nueva York: Springer, 2018, pp. 1013-1026. https:// doi.org/10.1007/978-3-319-72761-5_71

Waldenfels, B., Exploraciones fenomenológicas acerca de lo extraño, Barcelona/Morelia: Anthropos/UMSNH, 2015.

Zahavi, D., "Husserl's Intersubjective Transformation of Transcendental Philosophy", en: Welton, D. (ed). The New Husserl. A Critical Reader, Bloomington: Indiana University Press, 2003, pp. 233-251.

Zahavi, D., Subjectivity and Selfhood: Investigating the First-person Perspective, Cambridge, MA.: MIT University Press, 2006. https://doi.org/10.7551/ mitpress /6541.001.0001 